\title{
ANESTESIA EM EQÜINOS COM SÍNDROME CÓLICA - ANÁLISE DE 48 CASOS E REVISÃO DE LITERATURA
}

\author{
ANESTHESIA IN HORSES WITH COLIC SYNDROME - ANALYSIS OF 48 \\ CASES AND LITERATURE REVIEW
}

\author{
Alonso Gabriel Pereira Guedes ${ }^{1}$ Claudio Corrêa Natalini ${ }^{2}$
}

RESUMO

A cólica eqüina é uma síndrome que cursa com dor abdominal, distúrbios hidroeletrolíticos e ácido-base e disfunção de órgãos vitais como pulmões e coração. Os procedimentos anestésicos nesses animais apresentam particularidades que aumentam o risco de complicações. Os animais devem ser avaliados no período pré-anestésico e as terapias de reposição devem ser instituídas quando necessárias. A medicação préanestésica deve proporcionar analgesia e sedação do animal. A xilazina elou butorfanol podem ser utilizados com esse objetivo. A indução pode ser realizada com éter gliceril guaiacolato $e$ cetamina com ou sem diazepam, ou mesmo com cetamina $e$ diazepam pela via intravenosa. A manutenção anestésica deve ser feita preferencialmente com isofluorano, mas o halotano também pode ser utilizado. Manter ventilação pulmonar mecânica, com o animal recebendo oxigênio a $100 \%$ durante todo o período cirúrgico e pós-operatório imediato. A recuperação deve ser feita em ambiente escurecido e calmo, com forração e piso não escorregadio. Analgesia e oxigenoterapia também são importantes nessa fase.

Palavras-chave: anestesia, eqüino, cólica, xilazina, butorfanol, cetamina, halotano, isofluorano.

\section{SUMMARY}

The equine colic is a syndrome that leads to abdominal pain, hydroeletrolitic and acid-base disturbances and functional alterations of the vital organs like lungs and heart. Anesthetic procedures in these animals show certain particularities that elevate the complications risk. The animals should be evaluated at the preanesthetic period and the reposition treatments should be done as necessary. The premeds should give analgesia and sedation to the animal. Xylazina and/or butorphanol may be used to this aim. Induction can be done with guaiacol glycerine ether, ketamine with/without diazepam, or even with ketamine and diazepam by the intravenous route.
Isoflurane is the anesthetic of choice for the maintenance of the anesthesia, but halothane also can be used. Intermitent positive pressure ventilation should be established, with the animal receiving $100 \%$ oxygen throughout the surgical period and the imediate postoperatory period. Recovery should be done in a darkness and calm environment, with soft and not slippery floor. Analgesia and oxygen therapy also are important during this stage.

Key words: anesthesia, equine, colic, xylazine, butorphanol, ketamine, halothane, isoflurane.

\section{INTRODUÇÃO}

O manejo clínico, cirúrgico e anestésico de eqüinos com doenças abdominais tem melhorado marcadamente (HODGSON \& DUNLOP, 1990; MEE $\boldsymbol{e t}$ al., 1998). Eqüinos com síndrome cólica submetidos à cirurgia de emergência podem ter compromisso cardiopulmonar de moderado a severo (HODGSON \& DUNLOP, 1990), possuindo um risco de mortalidade de 9,86 vezes maior que em casos eletivos (MEE $\boldsymbol{e t}$ al., 1998). Existe uma alta incidência de óbitos durante a indução e manutenção anestésicas e no período de recuperação, pela inabilidade de levantar, incluindo miopatia pósanestésica e fraturas de ossos longos. Ocorre ainda uma alta incidência de choque pós-cirúrgico e colapso cardiovascular que podem, inclusive, incidir muitos dias após a anestesia (HODGSON \& DUNLOP, 1990).

Muitas combinações anestésicas podem ser utilizadas com sucesso em doenças específicas,

\footnotetext{
${ }^{1}$ Médico Veterinário, aluno do Programa de Pós-graduação em Medicina Veterinária, Universidade Federal de Santa Maria (UFSM),

97105-900, Santa Maria, RS. E-mail: aguedes@lince.hcv.ufsm.br. Autor para correspondência.

${ }^{2}$ Médico Veterinário, Mestre, PhD., Professor, Departamento de Clínica de Pequenos Animais, (UFSM).
}

Recebido para publicação em 09.02.01. Aprovado em 01.08.01 
procedimentos diagnósticos ou cirurgias em eqüinos. Para que se possa prover um cuidado anestésico adequado deve-se: 1) avaliar o paciente quanto à presença de alterações fisiológicas; 2) promover suporte pré-anestésico (fluidoterapia, expansores plasmáticos, analgésicos, antiinflamatórios, etc.) e, em alguns casos, tratamentos que irão minimizar os efeitos dessas alterações; 3) elaborar um plano anestésico que irá minimizar disfunções fisiológicas; 4) realizar monitoração intraoperatória e cuidados de suporte (fluidoterapia, expansores plasmáticos, analgésicos, inotrópicos positivos e/ou vasopressores); e 5) continuar com monitoração, de acordo com a necessidade, no período de recuperação e pós-recuperação. Independentemente dos anestésicos ou dos fármacos utilizados, a monitoração da resposta do paciente ao procedimento anestésico-cirúrgico e o ajuste da dose anestésica quando necessária são essenciais para uma ótima recuperação (HODGSON \& DUNLOP, 1990).

HODGSON \& DUNLOP (1990) citam que ainda são limitadas as informações a respeito das alterações cardiopulmonares pré-existentes em pacientes saudáveis ou comprometidos, submetidos à anestesia e cirurgia. REIMER et al. (1992) reportam que, de um grupo de 21 eqüinos com arrtimias ventriculares, 12 tinham doença sistêmica concomitante, sendo sete com desordem do trato gastrintestinal. Para HODGSON \& DUNLOP (1990), pressão arterial sistêmica, hemogasometria arterial e pH são comumente mensurados em eqüinos submetidos à cirurgia abdominal. Entretanto, débito cardíaco, distribuição do fluxo sangüíneo e volume intravascular são desconhecidos. Presume-se que muitos desses pacientes têm volume intravascular e débito cardíaco reduzidos e que a anestesia e o decúbito irão deprimir ainda mais a função cardiopulmonar numa magnitude maior que em animais saudáveis anestesiados. A mensuração do hematócrito e das proteínas plasmáticas totais tanto antes quanto durante o procedimento anestésico dão importantes informações acerca do manejo anestésico a ser empregado.

A presente revisão de literatura tem por objetivo reunir as informações existentes sobre o assunto e discuti-las, bem como apresentar dados de casos clínicos, servindo como fonte de consulta a estudantes e profissionais.

\section{RELATO DOS CASOS}

Os procedimentos anestésicos discutidos neste artigo foram realizados em eqüinos pacientes da clínica de grandes animais da Universidade de Minnesota-EUA. Foram analisadas 48 fichas anestésicas de eqüinos com síndrome cólica submetidos a tratamento cirúrgico, no período de 1997 a 1999. Eram todos animais adultos, machos ou fêmeas, pertencentes a diferentes raças, pesando entre $363 \mathrm{~kg}$ e $725 \mathrm{~kg}$.

Xilazina, butorfanol ou ambos foram utilizados na medicação pré-anestésica. Para a indução anestésica, foram empregadas as combinações éter gliceril guaiacolato (EGG) e cetamina em 23 animais (48\%) ou EGG, cetamina e diazepam em 25 animais (52\%). Três animais (12\%) que tiveram a anestesia induzida com EGG, cetamina e diazepam necessitaram de uma suplementação com tiopental sódico para que a indução fosse definitivamente efetivada. Dos animais cuja anestesia foi induzida com EGG e cetamina, três (13\%) foram suplementados com cetamina, dois $(8,7 \%)$ com cetamina e diazepam e um $(4,3 \%)$ com tiopental e cetamina para que se efetivasse a indução anestésica. A manutenção anestésica foi realizada com isofluorano $(85,4 \%$ dos animais) ou halotano (14,6\% dos animais), administrados em oxigênio através de sistema anestésico semi-fechado, com ventilação controlada por pressão positiva intermitente (VPPI) desde o início da anestesia.

Todos os animais tiveram monitoradas as pressões arteriais sistólica, média e diastólica, o ritmo cardíaco e as freqüências cardíaca e respiratória a intervalos de cinco minutos. Avaliação hemogasométrica arterial bem como mensuração da concentração plasmática de sódio e potássio foram realizadas em 34 animais. No período intraoperatório, os fármacos de suporte cardiovascular, os anestésicos intravenosos, os analgésicos e/ou antiinflamatórios (Tabela 1) foram utilizados de acordo com a necessidade.

Hipotensão (PAM $<70 \mathrm{mmHg}$ ) temporária (10 a 25 minutos) ou persistente (30 minutos ou mais) foi observada em $41,6 \%$ e $29,2 \%$ dos animais, respectivamente. $\mathrm{O}$ grupo de animais cuja anestesia foi induzida com EGG, cetamina e diazepam teve 16 animais $(64 \%)$ com hipotensão (em 12 temporária e em quatro persistente) transoperatória. Somente um desses animais, com hipotensão temporária, não recebeu medicação de suporte cardiovascular. Dos animais cuja anestesia foi induzida com EGG e cetamina, 18 apresentaramse hipotensos $(78,3 \%)$, sendo oito de forma temporária e 10 de forma persistente. Desses, apenas dois animais (um com hipotensão temporária e outro persistente) não foram medicados com fármacos de suporte cardiovascular (Tabela 2). Quatorze animais 
Tabela 1 - Fármacos intravenosos utilizados no período transoperatório em 48 eqüinos com síndrome cólica.

\begin{tabular}{|c|c|}
\hline Fármacos intravenosos transoperatórios & $\mathrm{N}^{\mathrm{o}}$ de animais \\
\hline \multicolumn{2}{|l|}{ Fluidoterapia } \\
\hline Ringer lactato de sódio & 48 \\
\hline \multicolumn{2}{|l|}{ Suporte cardiovascular } \\
\hline Dobutamina & 33 \\
\hline Dobutamina e salina hipertônica & 04 \\
\hline Outros fármacos ou combinações & 06 \\
\hline Nenhum & 05 \\
\hline \multicolumn{2}{|l|}{ Anestésicos ou analgésicos intravenosos } \\
\hline Cetamina e diazepam & 07 \\
\hline Cetamina e xilazina & 08 \\
\hline Cetamina e butorfanol & 04 \\
\hline Cetamina & 08 \\
\hline Outros fármacos ou combinações & 07 \\
\hline Nenhum & 14 \\
\hline \multicolumn{2}{|l|}{ Reposição eletrolítica } \\
\hline Bicarbonato de sódio & 01 \\
\hline Cloreto de potássio & 01 \\
\hline Cloreto de potássio e bicarbonato de sódio & 01 \\
\hline Bicarbonato de sódio e gluconato de cálcio & 01 \\
\hline
\end{tabular}

$(29,2 \%)$, dos quais nove $(18,75 \%)$ tiveram a anestesia induzida por EGG, cetamina e diazepam, apresentaram-se normotensos durante todo o procedimento, sem que fosse necessária medicação inotrópica positiva.

A maioria dos episódios de hipotensão foram observadas nos primeiros vinte minutos de monitoração da pressão arterial. A causa foi provavelmente a ação dos fármacos utilizados para a indução da anestesia, com alguma contribuição daqueles usados na MPA, especialmente pela ação vasodilatadora da xilazina, que poderia estar ocorrendo. Observaram-se índices de 78,3\% de hipotensão inicial dentre os animais cuja anestesia foi induzida com EGG e cetamina. Quando o diazepam foi incluso na indução anestésica, apenas $44 \%$ dos animais apresentaram-se inicialmente hipotensos. Contudo, essa é apenas uma constatação, sendo difícil explicá-la pois são casos clínicos com muitas variáveis, as quais não estavam sob controle e podem ter influenciado esse resultado. A resposta para essa dúvida talvez possa ser obtida com a realização de um experimento em modelo experimental padronizado, com um maior controle das variáveis.

\section{AVALIAÇÃo PRÉ-ANESTÉSICA}

Os desequilíbrios eletrolíticos devem ser corrigidos sempre que necessário, com base nos valores do $\mathrm{pH}$ sangüíneo e do $\mathrm{CO}_{2}$ total (HODGSON \& DUNLOP, 1990). A quantidade de bicarbonato a ser administrada é obtida através da fórmula: Peso $(\mathrm{kg})$ x $0,3(\ell / \mathrm{kg}) \times$ déficit de base $(\mathrm{mEq} / \ell)=\mathrm{NaHCO}_{3}$ requerido (mEq) (DAUNT, 1990; SEELER, 1996). HODGSON \& DUNLOP (1990) enfatizam que durante a administração deve ser realizada uma série de determinações do $\mathrm{pH}$ e $\mathrm{CO}_{2}$ total ou, se possível, do bicarbonato sérico para acessar a resposta à terapia. A síndrome cólica, quando decorrente de complicações no trato digestivo, pode levar à hipocalemia por perda de potássio para a luz intestinal. Assim, a concentração sérica de potássio também deve ser avaliada (SEELER, 1996), devendo ser corrigida sempre que houver redução aguda para 2,5 a $3,0 \mathrm{mEq} / \ell$. O cloreto de potássio pode ser infundido numa velocidade não superior a $0,5 \mathrm{mEq} / \mathrm{kg} / \mathrm{h}$, com dose máxima diária de 2 a $3 \mathrm{mEq} / \mathrm{kg}$. O paciente deve ser monitorado continuamente durante esse período, inclusive com dosagens do potássio plasmático e com eletrocardiograma para detecção precoce dos sinais de toxicidade.

A avaliação hemogasométrica informará sobre a oxigenação sanguiínea (tensão de oxigênio arterial $-\mathrm{PaO}_{2}$ ) e a severidade da dificuldade ventilatória (tensão de gás carbônico arterial$\left.\mathrm{PaCO}_{2}\right)$. Se existir evidência de hipoxemia (membranas mucosas cianóticas ou $\mathrm{PaO}_{2}$ inferior a $70 \mathrm{mmHg}$ ) durante o exame pré-anestésico, indução anestésica ou recuperação pós-anestésica, deve-se administrar oxigênio suplementar ao paciente (HODGSON \& DUNLOP, 1990).

\section{MEDICAÇÃO PRÉ-ANESTÉSICA}

Xilazina $(35,41 \%)$ ou xilazina e butorfanol $(33,33 \%)$ foram os fármacos mais freqüentemente utilizados na MPA dos animais. Em 27,1\% deles, não foi necessário administrar MPA pois já se encontravam sedados pela medicação que receberam quando estavam sob cuidados da clínica médica. É sempre importante que o anestesista obtenha essas informações pois, conforme HODGSON \& DUNLOP (1990), eqüinos com dor abdominal recebem altas doses de sedativos ou fármacos analgésicos, incluindo xilazina e butorfanol, antes da indução anestésica. Maior sedação geralmente não se faz necessária mas, caso for, devem ser usadas pequenas doses de analgésicos ou sedativos (butorfanol $0,02 \mathrm{mg} / \mathrm{kg} / \mathrm{IV}$, ou xilazina $0,1-0,2 \mathrm{mg} / \mathrm{kg} / \mathrm{IV})$. A xilazina é um potente analgésico com excelente analgesia visceral (THURMON \& BENSON, 1987), sendo utilizada no controle da dor na cólica equiina (HODGSON \& 
Tabela 2 - Fármacos de suporte cardiovascular utilizados no período transoperatório em 48 eqüinos com síndrome cólica, de acordo com o protocolo de indução anestésica.

\begin{tabular}{llcc}
\hline \multirow{2}{*}{$\begin{array}{l}\text { Indução } \\
\text { anestésica }\end{array}$} & \multicolumn{1}{c}{ Suporte cardiovascular } & \multicolumn{2}{c}{$\mathrm{N}^{\mathrm{o}}$ de animais em hipotensão (*) } \\
\cline { 3 - 4 } & & Temporária & Persistente \\
\hline & Dobutamina & 10 & 03 \\
EGG, cetamina & Hetastarch & 01 & 01 \\
e diazepam & Nenhuma & & \\
Sub-total & & 01 & 04 \\
& Dobutamina dobutamina e dopamina & 12 & 07 \\
EGG e cetamina & Nenhuma & 06 & 01 \\
& Efedrina e salina hipertônica & 01 & 01 \\
& Efedrina, dobutamina, hetastarch, & 01 & \\
dextran 70 e salina hipertônica & & 01 \\
Sub-total & Dobutamina e salina hipertônica & & 10 \\
TOTAL & & 08 & 14 \\
\hline
\end{tabular}

(*) Considerou-se hipotensão uma pressão arterial média inferior a 70mmHg. Temporária quando se manteve por um período entre 10 e 25 minutos e persistente quando igual ou superior a 30 .minutos.

Em eqüinos saudáveis, a administração intravenosa de xilazina, detomidina, medetomidina ou romifidina produz hipertensão inicial transitória dose-dependente (ENGLAND \& CLARKE, 1996), seguida por leve, porém, prolongada redução na pressão arterial mesmo com o uso de pequenas doses.

O butorfanol produz menor grau de analgesia que a xilazina mas possui a vantagem de causar menor depressão cardiovascular (HODGSON \& DUNLOP, 1990). Ainda, causa mínimos efeitos cardiovasculares nas doses comumente utilizadas (THURMON \& BENSON, 1987). O butorfanol tem sido associado aos agonistas alfa $_{2}$ adrenérgicos (ENGLAND \&

DUNLOP, 1990; ENGLAND \& CLARKE, 1996). Também causa redução na motilidade intestinal (ENGLAND \& CLARKE, 1996; HODGSON \& DUNLOP, 1990) sendo possível que isso possa contribuir com seu o efeito analgésico (ENGLAND \& CLARKE, 1996).

Os fármacos alfa $_{2}$ agonistas (xilazina, romifidina e detomidina) causam bradicardia, comumente acompanhada por bloqueio atrioventricular (ENGLAND \& CLARKE, 1996; HODGSON \& DUNLOP, 1990), bloqueio sinoatrial menos freqüentemente (THURMON \& BENSON, 1987; ENGLAND \& CLARKE, 1996) e redução no débito cardíaco (HODGSON \& DUNLOP, 1990). $\mathrm{Na}$ maioria dos animais, os bloqueios são mais intensos nos primeiros minutos após a administração. Após esse período, a freqüência cardíaca eleva-se gradativamente e os bloqueios desaparecem. A bradicardia e os bloqueios cardíacos persistem por mais tempo após administração de romifidina do que com xilazina ou detomidina (ENGLAND \& CLARKE, 1996). A detomidina tem ação similar à xilazina, mas é 10 vezes mais potente e tem ação mais prolongada, devendo ser utilizada com cuidado em eqüinos comprometidos. Em segmentos intestinais isolados, a xilazina causa aumento na resistência vascular e aumenta o consumo de oxigênio que pode ser ainda mais importante se a perfusão intestinal já estiver comprometida (HODGSON \& DUNLOP, 1990).
CLARKE, 1996; HUBBELL, 1996), não alterando a função cardiovascular além daquela causada pelo alfa $_{2}$ agonista, embora possa haver um leve incremento na depressão respiratória. Alguns cavalos podem movimentar-se para frente compulsivamente e exibir tremores musculares, efeitos atribuídos ao componente opióide dessas combinações (ENGLAND \& CLARKE, 1996). Esse efeito não foi observado nos dados coletados das fichas dos animais nos quais o butorfanol foi utilizado. Contudo, essa combinação é benéfica dada a possibilidade de redução na dose da xilazina (HUBBELL, 1996), e, por conseguinte, dos efeitos indesejáveis de doses maiores de alfa ${ }_{2}$ agonistas (ENGLAND \& CLARKE, 1996).

\section{INDUÇÃO DA ANESTESIA}

A indução anestésica em eqüinos apresentados para cirurgia abdominal de emergência pode ser realizada com éter gliceril guaiacolato $(100 \mathrm{mg} / \mathrm{kg} / \mathrm{IV})$ seguida por bolus de cetamina (2mg/kg/IV) (HODGSON \& DUNLOP, 1990). Em equiinos sedados com xilazina (BROCK \& HILDEBRAND, 1990), o diazepam $(0,1 \mathrm{mg} / \mathrm{kg})$ pode substituir o EGG nas combinações com cetamina, promovendo indução, transição para a anestesia inalatória e recuperação anestésica similares. Após avaliar cinco combinações farmacológicas para indução anestésica, WAGNER 
et al. (1996) concluíram que o protocolo de indução tem pouco impacto na função hemodinâmica de equiinos saudáveis anestesiados com halotano.

Os dados obtidos das fichas clínicas mostraram a utilização de duas técnicas de indução, uma composta por EGG, cetamina e diazepam e outra por EGG e cetamina. Realizou-se um tratamento estatístico (ANOVA com medidas repetidas e teste de Tukey com nível de significância de $5 \%$ ) entre os dois grupos de animais. Foram excluídos os animais que receberam doses suplementares de anestésicos intravenosos na transição para a anestesia inalatória. Dessa forma, 17 animais formaram o grupo cuja anestesia foi induzida com EGG e cetamina (GC) e 19 com EGG, cetamina e diazepam (GCD). Na manutenção anestésica, empregaram-se halotano em três animais do grupo GC e dois do grupo GCD e isofluorano nos demais. Foram avaliadas a freqüência cardíaca (FC), as pressões arteriais sistólica (PAS), média (PAM), diastólica (PAD) e a concentração de anestésico inalatório emitida pelo vaporizador (V\%). As verificações foram feitas a cada 15 minutos, a partir do início da cirurgia, durante o período cirúrgico máximo de 120 minutos.

As médias das variáveis PAM e PAD foram significativamente inferiores no grupo GC $(\mathrm{p}<0,05)$, mas clinicamente aceitáveis. A V\% sofreu redução significativa $(\mathrm{p}<0,05)$ com o avanço do período de observação dentro do grupo GCD, mas não no grupo GC. As demais variáveis não mostraram qualquer diferença estatisticamente significativa. Embora essas informações tenham valor limitado por causa da falta de controle de uma série de variáveis, pode-se afirmar que a inclusão do diazepam na indução anestésica de eqüinos com síndrome cólica não afeta clinicamente as variáveis hemodinâmicas. Com base nesses resultados e na experiência clínica, indica-se a inclusão do diazepam nas induções anestésicas de eqüinos com síndrome cólica realizadas com EGG e cetamina.

\section{MANUTENÇÃO ANESTÉSICA}

HODGSON \& DUNLOP (1990) relatam que tanto halotano quanto isofluorano em oxigênio são utilizados para a manutenção anestésica em eqüinos com síndrome cólica. Ambos os agentes causam depressão cardiopulmonar dose-dependente (STEFFEY, 1978; STEFFEY \& HOWLAND, 1980; HUBBELL, 1996; GROSENBAUGH \& MUIR, 1998) em eqüinos saudáveis. O débito cardíaco é mantido melhor com o isofluorano em relação ao halotano (STEFFEY \& HOWLAND, 1980; GROSENBAUGH \& MUIR, 1998), o que pode ser vantajoso em pacientes comprometidos (HODGSON \& DUNLOP, 1990). HARVEY et al. (1987) observaram poucas diferenças com o uso desses dois anestésicos em eqüinos com cólica, exceto um menor tempo de recuperação dos animais anestesiados com isofluorano. GROSENBAUGH \& MUIR (1998) afirmam que o sevofluorano apresenta efeitos hemodinâmicos, respiratórios e no tempo de recuperação anestésica similares ao isofluorano, apenas possibilitando um melhor controle da profundidade anestésica.

Nos casos clínicos aqui relacionados, a vaporização do anestésico inalatório foi ajustada conforme necessário para manter um plano cirúrgico de anestesia (HARVEY et al., 1987; WAGNER $\boldsymbol{e t}$ al., 1996). Apesar da não mensuração da concentração alveolar dos anestésicos, os resultados da análise dos dados clínicos sugerem que a inclusão do diazepam no protocolo de indução anestésica formado por EGG e cetamina, pode reduzir o consumo de anestésico inalatório. Uma possível explicação para esse fato é o relaxamento muscular e o efeito hipnótico adicionais produzidos pelo diazepam (BROCK \& HILDEBRAND, 1990) podendo ter levado a uma menor necessidade de anestésico inalatório para a manutenção do plano de anestesia cirúrgica. $O$ valor dessa redução é enfatizado por THURMON (1990), quando diz que, além do tempo de anestesia, a manutenção da menor profundidade anestésica possível é muito importante para reduzir as complicações pós-anestésicas em equiinos adultos.

A recumbência faz com que a oxigenação sangüínea seja afetada significativamente com algum grau de hipoxemia ocorrendo em quase todos os animais (THURMON, 1990, HUBBELL, 1996), sendo mais severa naqueles posicionados em decúbito dorsal (STEFFEY et al., 1977; DAY et al., 1995). Além disso, o timpanismo abdominal (HODGSON \& DUNLOP, 1990) pode causar disfunção cardiopulmonar severa, resultando em trocas gasosas deficientes e inabilidade de expandir os pulmões adequadamente. O decúbito dorsal (STEFFEY et al., 1977; THURMON, 1990; DAY $\boldsymbol{e}$ t al., 1995), o peso elevado e a compressão diafragmática pelas vísceras abdominais (THURMON, 1990) podem causar hipoxemia transoperatória, acidose respiratória e hipercapnia, conforme observado em um dos animais do presente levantamento. Esse apresentava cólica por retenção fetal, o qual, juntamente com as demais vísceras abdominais, pressionava a cúpula diafragmática, levando à compressão e atelectasia dos lobos pulmonares caudais (DAY et al., 1995). Nesse animal, nem mesmo o uso de ventilação com pressão 
positiva intermitente (VPPI) com volume corrente de 12 litros e pressão inspiratória de $45 \mathrm{cmH}_{2} \mathrm{O}$ foi suficiente para reverter o quadro respiratório. Nesses casos, DAY et al. (1995) explicam que a VPPI realmente pode ser ineficaz, provavelmente, por não poder gerar uma pressão suficiente para expandir os pulmões atelectásicos. Essas áreas são melhor ventiladas quando a VPPI é instituída antes de ocorrer atelectasia dos lobos pulmonares caudais mas, apesar disso, alguns cavalos desenvolvem hipoxemia mesmo durante a ventilação controlada.

Todos os animais do presente estudo tiveram a ventilação controlada com VPPI no intuito de otimizar a ventilação alveolar e, por conseguinte, melhorar as trocas gasosas dos pacientes. Apesar disso, alguns autores não estabelecem rotineiramente a VPPI logo após a indução anestésica de eqüinos submetidos a cirurgias gastrintestinais de emergência. Explicam que, embora possa haver incremento na $\mathrm{PaO}_{2}$ (HODGSON \& DUNLOP, 1990), esse modo de ventilação reduzirá ainda mais o débito cardíaco, pois a pressão positiva intratorácica prejudica o retorno venoso ao coração (HODGSON \& DUNLOP, 1990; KLEIN，1990; SHAWLEY \& MANDSAGER, 1990). Contudo, DAY et al. (1995) alertam para o fato de que após o desenvolvimento de hipoxemia durante ventilação espontânea, a VPPI melhora a oxigenação apenas nos animais posicionados em decúbito lateral, mas não naqueles em decúbito dorsal. Esse fato justifica a instalação da VPPI desde o início do procedimento anestésico, já que os animais são posicionados em decúbito dorsal. Além disso, a ocorrência de hipercapnia aumenta a possibilidade de arritmias cardíacas. SHAWLEY \& MANDSAGER (1990) dizem que a VPPI permite a manutenção dos valores dos gases sangüíneos arteriais próximos do normal com enchimento venoso normal da veia cava e átrio direito, mesmo durante procedimentos de longa duração. STEFFEY (1978) diz que a depressão cardiovascular causada pela VPPI é menor com isofluorano do que com halotano.

Sabendo que a VPPI pode prejudicar a função cardiovascular, essa variável deve ser constantemente monitorada e, quando não for possível prever e prevenir as alterações, essas devem ser corrigidas o quanto antes. A oxigenação sangüínea e o equilíbrio ácido-base também devem ser acessados através de hemogasometrias arteriais. A maioria dos pacientes com síndrome cólica necessitarão de terapia de suporte cardiovascular, conforme observado no presente levantamento. Dos 48 animais incluídos, apenas cinco não receberam esse tipo de terapia, a não ser fluidoterapia que foi instituída em todos os animais. Dentre os fármacos inotrópicos positivos utilizados, a dobutamina isoladamente em infusão contínua foi a mais utilizada (Tabela 1 ). Por ser um agonista $\beta_{1}$ seletivo nas doses utilizadas (1 a $5 \mu \mathrm{g} / \mathrm{kg} / \mathrm{min}$ ) (MUIR \& McGUIRK, 1987; DAUNT, 1990), a dobutamina possui a vantagem de elevar o débito cardíaco, não interferindo com a resistência vascular periférica (KLEIN, 1990), causando aumento no fluxo sangüíneo para a musculatura esquelética (MUIR \& McGUIRK, 1987). Essa característica é importante na prevenção de miopatias pós-anestésicas.

HODGSON \& DUNLOP (1990) argumentam que, se o paciente está hipotenso, pode ser vantajoso utilizar a cetamina como suplemento da anestesia para reduzir a dose de isofluorano ou halotano. A observação das fichas clínicas demonstrou que $64,6 \%$ dos animais receberam cetamina no período transanestésico, combinado ou não com diazepam, xilazina, butorfanol ou tiopental. Esses fármacos foram utilizados tanto para promoção de analgesia como para aprofundar o plano anestésico muito superficial de alguns animais, já que se buscava mantê-lo o mais superfícial possível. Essas manobras podem ser utilizadas durante a anestesia inalatória, inclusive para re-indução anestésica caso for necessária (GROSENBAUGH \& MUIR, 1998) resultando em mínimos efeitos cardiopulmonares. O butorfanol $(0,02 \mathrm{mg} / \mathrm{kg}) \quad$ (HODGSON \& DUNLOP, 1990; KLEIN, 1990) ou a xilazina $(0,1$ a $0,2 \mathrm{mg} / \mathrm{kg})$ (KLEIN, 1990) também podem ser administrados para suplementar a anestesia, embora o efeito seja exercido apenas após quatro a cinco minutos (HODGSON \& DUNLOP, 1990; KLEIN, 1990).

Nos animais que estão em um plano anestésico adequado, mas são hipotensos, recomenda-se o uso de infusão contínua de dopamina (HODGSON \& DUNLOP, 1990) ou dobutamina na dose de 5 a $10 \mu \mathrm{g} / \mathrm{kg} / \mathrm{min}$ para elevar a pressão sangüínea. $\mathrm{O}$ presente estudo demonstrou que raramente são necessárias doses maiores que 5 $\mu \mathrm{g} / \mathrm{kg} / \mathrm{min}$ para que a pressão arterial aumente. A efedrina $(0,03$ a $0,06 \mathrm{mg} / \mathrm{kg})$ pode também ser administrada para elevar o débito cardíaco e a pressão arterial (HODGSON \& DUNLOP, 1990; KLEIN, 1990). Comparada com a dopamina, a dobutamina é um $\beta_{1}$ agonista mais potente, com atividade inotrópica maior que a cronotrópica (DAUNT, 1990), tendo sido demonstrado ser mais eficaz na restauração do débito cardíaco e da pressão sangüínea sistêmica em eqüinos anestesiados com halotano. Ainda, MUIR \& McGUIRK (1987) dizem que a dobutamina é menos arritmogênica, tem menor potencial de induzir efeito epinefrínico

Ciência Rural, v. 32, n. 3, 2002. 
reverso e produz um melhor efeito que a dopamina sobre o volume ejetado e o débito cardíaco de animais com colapso circulatório cardiogênico.

Dos fluidos disponíveis, a solução de Ringer lactato é apropriada para a maioria dos casos. Nas obstruções do intestino grosso associadas com bicarbonato sérico maior que $30 \mathrm{mEq} / \ell$, está indicada solução salina a $0,9 \%$. Animais em hipovolemia severa podem beneficiar-se, inicialmente, da administração de salina hipertônica a $7 \%(4 \mathrm{~m} \ell / \mathrm{kg})$. Se a albumina plasmática for menor que 1,5 a $2,0 \mathrm{~g} / \mathrm{d} \ell$, ou a proteína total estiver abaixo de 3 a $4 \mathrm{~g} / \mathrm{d} \ell$, devem ser administrados fluidos colóides, para recuperar a pressão oncótica e evitar o desenvolvimento de edema pulmonar. Podem ser utilizados plasma ou expansores plasmáticos sintéticos como o dextran 40, dextran 70 e hetastarch quando o plasma não estiver disponível. A meia vida plasmática dos expansores plasmáticos sintéticos é de aproximadamente duas horas para o dextran 40, seis horas para o dextran 70 e hetastarch, enquanto que para o plasma é de 12 a 18 horas (DAUNT, 1990).

As arritmias cardíacas podem estar associadas a desordens gastrintestinais, podendo ser causadas por hipóxia, distúrbios metabólicos e eletrolíticos, toxinas e aos anestésicos inalatórios, ou alterações cardíacas primárias (REIMER, 1992). Neste levantamento, as arritmias cardíacas transoperatórias foram observadas raramente, correspondendo à contração atrial prematura em três animais e bloqueio atrioventricular de $2^{\circ}$ grau em outro após administração intra-operatória de xilazina. Essas arritmias resolveram-se sem a necessidade de intervenção farmacológica sendo que raramente necessitam de tratamento (ENGLAND \& CLARKE, 1996; KLEIN, 1990). A terapia deve ser considerada quando houver interferência hemodinâmica, com base no eletrocardiograma, exame físico, doença cardíaca e dados laboratoriais (REIMER, 1992).

\section{RECUPERAÇÃO ANESTÉSICA}

A recuperação das anestesias com halotano (HARVEY et al., 1987) ou isofluorano (STEFFEY, 1978; HARVEY et al., 1987) são normalmente tranqüilas em eqüinos acometidos por cólica. A recuperação deve ser em local escuro, silencioso, com piso não escorregadio (HODGSON \& DUNLOP, 1990; HUBBELL, 1996) e o tubo orotraqueal deve ser mantido até que o animal esteja em decúbito esternal ou mesmo em estação (HODGSON \& DUNLOP, 1990).

Conforme observado nas fichas clínicas, utilizou-se xilazina $(0,1$ a $0,3 \mathrm{mg} / \mathrm{kg})$ para melhorar a recuperação anestésica (THURMON \& BENSON, 1987; GROSENBAUGH, 1995). Esse fármaco produz analgesia e sedação, fazendo com que a passagem do estado de inconsciência para o estado de consciência seja mais tranqüilo. Deve-se sempre conferir a patência das vias respiratórias superiores, pois edema e congestão venosa podem contribuir para sua obstrução (KLEIN, 1990). Com isso em mente, procurou-se promover vasoconstrição (DAUNT, 1990) local com administração de fenilefrina $(5 \mathrm{mg}$ em $5 \mathrm{~m} \ell$ de solução fisiológica em cada narina) em 12 animais para evitar ou atenuar a formação de edema nasal. Adicionalmente, um tubo nasotraqueal foi posicionado em 14,6\% dos animais, os quais apresentavam edema generalizado na região cervical e cabeça para evitar obstrução das vias respiratórias.

Oxigênio suplementar era administrado através de uma válvula de demanda colocada na extremidade rostral do tubo endotraqueal conforme indicado por HODGSON \& DUNLOP (1990), num fluxo de 10 a $15 \ell /$ minuto em todos os animais. Uma outra forma de fazer essa oxigenoterapia é através de um tubo que deve ser inserido na extremidade caudal do tubo endotraqueal, utilizando-se o mesmo fluxo de oxigênio (HODGSON \& DUNLOP, 1990). Essa manobra deve ser feita em todos os animais já que a hipoxemia ocorre rotineiramente (KLEIN, 1990) nos animais respirando ar ambiente durante a recuperação anestésica.

De um modo geral, as complicações pósanestésicas foram irrisórias, sendo a mais importante um caso de paralisia hipercalêmica periódica que ocorreu em um animal Quarto de Milha. Trata-se de uma doença genética de animais dessa raça descendentes do reprodutor Impressive (KOLLIASBAKER, 1999). Observou-se paresia pós-anestésica, além de elevada concentração $(7,4 \mathrm{mEq} / \ell)$ do potássio sérico. Terapia com bicarbonato de sódio gluconato de cálcio foi instituída (KOLLIAS-BAKER, 1999), com completa recuperação do animal.

\section{CONCLUSÃO}

A anestesia de eqüinos com síndrome cólica caracteriza-se por sua complexidade, e o controle das disfunções hemodinâmicas causadas pela doença é fundamental para o sucesso anestésico e de todo o tratamento. O exame clínico e o auxílio laboratorial são importantes para o acesso à condição física do paciente e para a escolha da melhor conduta anestésica. Para isso, o reconhecimento das alterações fisiopatológicas, o conhecimento de fisiologia e farmacologia são indispensáveis ao anestesista. 
De um modo geral, a revisão da literatura, a avaliação dos casos clínicos e a experiência clínica com esses animais permite fazer algumas considerações acerca do manejo anestésico. Deve-se avaliar clinicamente os animais, principalmente as funções cardiovascular e respiratória (ausculta cardíaca, palpação do pulso arterial e tempo de reperfusão capilar, frequiência e padrão respiratório, coloração das mucosas visíveis), e o grau de hidratação. A medicação pré-anestésica pode ser realizada com xilazina e/ou butorfanol, produzindo analgesia e sedação. Éter gliceril guaiacolato, cetamina e diazepam é a melhor combinação farmacológica para a indução anestésica. $\mathrm{Na}$ manutenção da anestesia o isofluorano é o anestésico de escolha, devendo ser fornecido em oxigênio a $100 \%$.

Deve-se instituir ventilação mecânica para prevenir episódios de hipoxemia e/ou hipercapnia e a pressão arterial sistêmica deve ser monitorada. Como a avaliação do débito cardíaco e da resistência vascular periférica não podem ser monitoradas nesses animais, a avaliação do tempo de reperfusão capilar pode dar informações subjetivas da perfusão tecidual. Fármacos inotrópicos positivos devem ser utilizados sempre que a pressão arterial reduzir para menos de $70 \mathrm{mmHg}$, com preferência para a dobutamina, por elevar a pressão arterial sem interferir com a resistência vascular sistêmica. $\mathrm{Na}$ recuperação anestésica, prover oxigenoterapia e analgésicos, realizando-a em local calmo, com pouca luz e bem acolchoado.

\section{REFERÊNCIAS BIBLIOGRÁFICAS}

BROCK, N., HILDEBRAND, S.V. A comparison of xylazine-diazepam-ketamine and xylazine-guaifenesinketamine in equine anesthesia. Vet Surg, v.19, n.6, p.468-474, 1990 .

DAUNT, D.A. Supportive therapy in the anesthetized horse. Vet Clin North Am Equine Pract, v.6, n.3, p.557-574, 1990

DAY, T.K., GAYNOR, J.S., MUIR, W.W., et al. Blood gas values during intermitent positive pressure ventilation and spontaneous ventilation in 160 anesthetized horses positioned in lateral or dorsal recumbency. Vet Surg, v.24, n.2, p.266$276,1995$.

ENGLAND, G.C.W., CLARKE, K.W. Alpha 2 adrenoceptor agonists in the horse - a review. Br Vet J, v.152, n.6, p.641$652,1996$.

GROSENBAUGH, D.A., MUIR, W.W. Cardiorespiratory effects of sevoflurane, isoflurane and halotane anesthesia in horses. Am J Vet Res, v.59, n.1, p.101-106, 1998.

HARVEY, R.C., GLEED, R.D., MATTHEWS, N.S., et al.
Isoflurane anesthesia for equine colic surgery - comparison with halothane anesthesia, Vet Surg, v.16, n.2, p.184-188, 1987.

HODGSON, D.S., DUNLOP, C.I. General anesthesia for horses with specific problems. Vet Clin North Am Equine Pract, v.6, n.3, p.625-650, 1990.

HUBBELL, J.A.E. Anesthesia and immobilization of specific species - Horses. In: THURMON, J.C., TRANQUILLI, W.J., BENSON, G.J. Lumb \& Jones' veterinary anesthesia. 3ed. Philadelphia : Williams \& Wilkins, 1996. Cap.20a, p.591-609.

KLEIN, L. Anesthetic complications in the horse. Vet Clin North Am Equine Pract, v.6, n.3, p.665-692, 1990.

KOLLIAS-BAKER, C. Therapeutics of musculoskeletal disease in the horse. Vet Clin North Am Equine Pract, v.15, n.3, p.589-602, 1999.

MEE, A.M., CRIPPS, P.J., JONES, R.S. A retrospective study of mortality associated with general anaesthesia in horses: emergency procedures. Vet Rec, v.142, n.3, p.307-309, 1998.

MUIR, W.W., McGUIRK, S. Cardiovascular drugs. Vet Clin North Am Equine Pract, v.3, n.1, p.37-57, 1987.

REIMER, J.M., REEF, V.B., SWEENEY, R.W. Ventricular arrhytmias in horses: 21 cases (1984-1989). J Am Vet Med Assoc, v.201, n.8, p.1237-1243, 1992

SEELER, D.C. Fluid and electrolite therapy. In: THURMON, J.C., TRANQUILLI, W.J., BENSON, G.J. Lumb \& Jones' veterinary anesthesia. 3ed. Philadelphia : Williams \& Wilkins, 1996. Cap.19, p.572-589.

SHAWLEY, R.V., MANDSAGER, R.E. Clinical use of positivepressure ventilation in the horse. Vet Clin North Am Equine Pract, v.6, n.3, p.575-585, 1990.

STEFFEY, E.P. Enflurane and isoflurane anesthesia: A summary of laboratory and clinical investigation in horses. J Am Vet Med Assoc, v.172, n.3, p.367-373, 1978.

STEFFEY, E.P., HOWLAND, D. Comparison of circulatory and respiratory effects of isoflurane and halothane anesthesia in horses. Am J Vet Res, v.41, n.5, p.821-825, 1980.

STEFFEY, E.P., WHEAT, J.D., MEAGHER, D.M., et al. Body position and mode of ventilation influences arterial $\mathrm{pH}$, oxygen, and carbon dioxide tensions in halothaneanesthetized horses. Am J Vet Res, v.38, n.3, p.379-382, 1977.

THURMON, J.C. General clinical considerations for anesthesia of the horse. Vet Clin North Am Equine Pract, v.6, n.3, p.485-494, 1990.

THURMON, J.C., BENSON, G.J. Injectable anesthetics and anesthetic adjuncts. Vet Clin North Am Equine Pract, v.3, n.1, p.15-36, 1987.

WAGNER, A.E., DUNLOP, C.I., WERTZ, E.M., et al. Evaluation of five common induction protocols by comparison of hemodynamic responses to surgical manipulation in halothane-anesthetized horses, J Am Vet Med Assoc, v.208, n.2, p.252-257, 1996. 